\title{
Acute portal vein thrombosis simulating acute pancreatitis: about a clinical case at the National University Hospital of Cotonou
}

\begin{abstract}
Introduction: Portal vein thrombosis (PVT) is a rare disease in the absence of cirrhosis Its clinical signs are non-specific and depend on when the diagnosis is made. The clinical case we are reporting highlights the possibility of diagnostic wandering, the contribution of imaging and the value of systematic etiological assessment.

Observation: We report here a 49-year-old patient with a large uterine myoma. She had consulted for a very intense transfixing epigastric pain, aggravated by meals and slightly relieved by fasting. An upper digestive endoscopy performed a few days after the beginning had revealed very intense erythematous gastritis. Lipasemia was normal. In view of the persistence of the symptoms despite appropriate treatment of gastritis, an abdominal CT scan concluded that there was a portal vein thrombosis. The etiological assessment did not show arguments in favour of cirrhosis, hepatocarcinoma, pancreatitis or pancreatic adenocarcinoma, but revealed HIV infection. The evolution was quickly favourable under anticoagulant.
\end{abstract}

Conclusion: Although non-specific, abdominal pain is the most common symptom of acute PVT. The diagnosis is based on the Doppler ultrasound and/or abdominal CT. The presence of a local or regional factor should not exclude the search for general causes such as HIV infection.

Keywords: portal vein thrombosis, epigastric pain, HIV infection, anticoagulation
Volume 10 Issue $6-2019$

\author{
Adjagba PM,' Kpossou AR, ${ }^{2}$ Adjadohoun \\ SBMG, ${ }^{3}$ Falolou FD,' Hounkponou M,' \\ Sonou A,' Tchabi Y,' Yèkpè P,' Sèhonou J,' \\ Biaou O, ${ }^{3}$ Houenassi DM' \\ 'Department of Cardiology, University of Abomey-Calavi (UAC), \\ Benin \\ ${ }^{2}$ Department of Hepato-Gatroenterology, Hubert Koutoukou \\ Maga National University Hospital Center, Benin \\ ${ }^{3}$ Department of Radiodiagnostic, Hubert Koutoukou Maga \\ National University Hospital Center, Benin
}

\begin{abstract}
Correspondence: Philippe Mahouna Adjagba, University Clinic of Cardiology, Hubert Koutoukou Maga National and University Hospital (CNHU-HKM), Cotonou, University of Abomey-Calavi (UAC), Benin, Tel +22997243282, Email dotup@yahoo.fr
\end{abstract}

Received: November 19, 2019 | Published: December 06, 2019

\begin{abstract}
Abbreviations: PVT, portal vein thrombosis; HIV, human immunodeficiency virus, CT, computerized tomography;
\end{abstract}

\section{Introduction}

Portal vein thrombosis (PVT) is the obstruction of the trunk of the portal vein by a blood clot. It can extend downstream into the intrahepatic branches of the portal vein or upstream into the splenic and/or mesenteric veins. The occlusion of the portal vein by tumor invasion or tumor compression with secondary development of a blood clot is also grouped under the name PVT. ${ }^{1}$ It is a rare disease in the absence of cirrhosis with an incidence of $0.7 / 100,000$ inhabitants in Europe. ${ }^{2,3}$ Cirrhosis is associated with increased intra-hepatic vascular resistance and reduced portal blood flow into the liver. Low portal blood flow seems to be the most important risk factor for PVT in cirrhosis and has been found to be predictive of future PVT. Apart from cirrhosis, multiple risk factors are identified and can be linked. Etiological factors are local or systemic. PVT may occur acutely or be identified only in the chronic phase. In acute obstruction, symptomatology can be highly variable, ranging from asymptomatic to dramatic intestinal ischemia. ${ }^{4}$ The non-specificity of the clinical picture may be a source of diagnostic wandering. It requires a rigorous diagnostic approach. ${ }^{4,5}$ We report here a clinical case highlighting this possibility of diagnostic wandering, the contribution of imaging and the value of systematic etiological assessment.

\section{Observation}

The patient was a 49-year-old with a history of uterine myoma. The onset of the symptomatology was one month ago with epigastric pain of $8 / 10$ intensity on the simple verbal scale, aggravated by food intake and partially relieved by the gun dog position. In response to this pain, the patient consulted a private clinic where, after an esophageal-gastro-duodenal endoscopy, she was diagnosed with erythematous gastritis. It should be noted that this endoscopy did not show any sign of portal hypertension, in particular any esophageal varix. She was therefore treated with a proton pump inhibitor and an antacid, but without success. Faced with the persistence of epigastric pain, she was referred for hospitalization at the University clinic of Hepato-Gastroenterology of the Hubert Koutoukou Maga National and University Hospital (CNHU/HKM) in Cotonou.

The physical examination found a mucocutaneous pallor, defenseless epigastric pain and a smooth, median pelvic rounded mass measuring $12 \mathrm{~cm}$ in diameter. This clinical presentation initially suggested acute pancreatitis. The lipasemia test then performed was normal. An abdominal CT without and with injection of iodized contrast agent (Figures 1-3) was therefore requested. This CT did not show any sign in favour of pancreatitis but rather a portal vein thrombosis in the form of an increase in the size of the portal trunk, splenomesaraïque trunk and splenic vein, spontaneously dense, not opacified on images with contrast agent injection, with a densification of the mesenteric fat. Collateral venous circulation was associated. The etiological assessment did not note any argument for cirrhosis (normal prothrombin rate at $100 \%$, normal albuminemia at $38 \mathrm{~g} / 1$, normal platelet rate at $156 \mathrm{Giga} / 1$, FIBROSIS-4 score at 1.9 , homogeneous liver not dysmorphic on ultrasound and abdominal CT), but showed positive serology for immunodeficiency virus 1 (HIV1). The diagnosis was an acute portal vein thrombosis extended to the splenic vein. The etiological factors were HIV infection and a large polymyomatous uterus. 


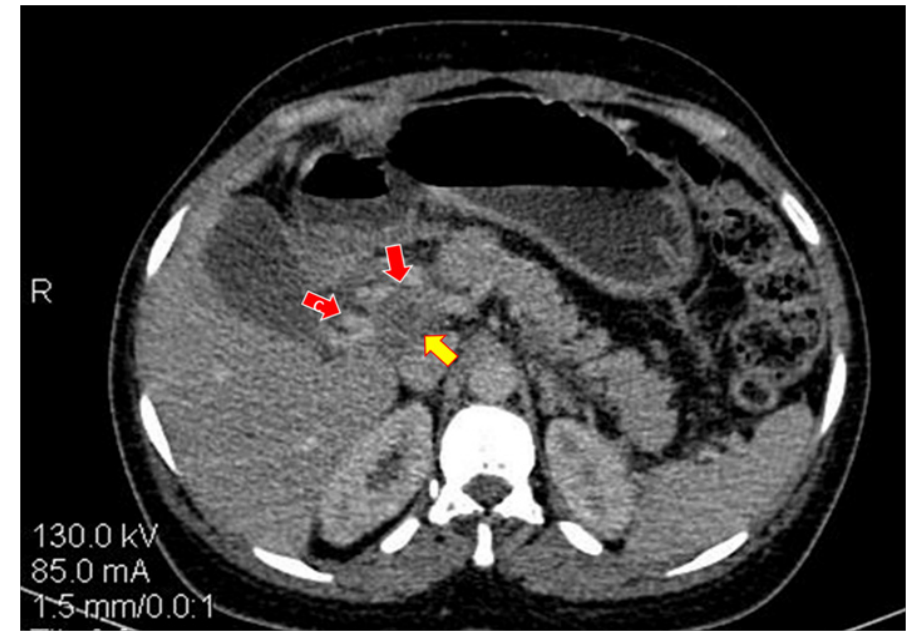

Figure I Abdominal-pelvic scanner with portal time injection.

Portal thrombosis in the form of swelling with hypodensity of the portal trunk (yellow arrow); portal carvernoma (collateral venous circulation designated by the red arrows).

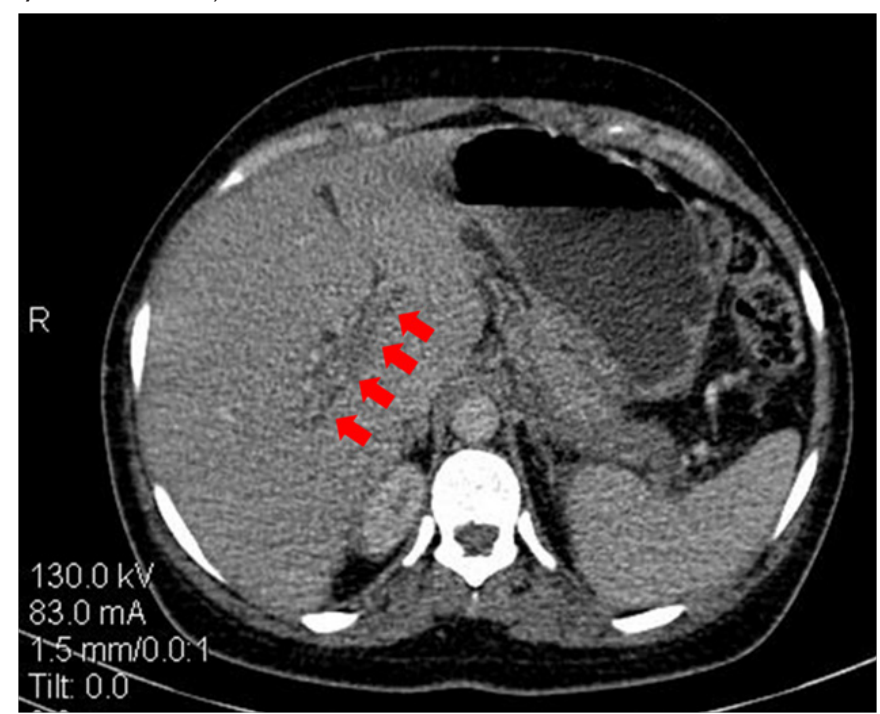

Figure 2 Abdominal-pelvic CT scan with portal time injection showing thrombosis of the right door branch.

The patient was therefore transferred to the cardiology department of CNHU/HKM where the treatment initiated in the clinic of Hepatogastroenterology with complete dietary restriction, strong opioid analgesia, and proton pump inhibitor was continued. In addition, a curative dose of enoxaparin anticoagulant was given.

The evolution was rapidly favourable with recession in epigastric pain after 48 hours of anticoagulation, allowing the analgesic to be stopped and the gradual resumption of feeding. Then we replaced enoxaparin with oral treatment with rivoxaban. The patient was able to return home on the thirteenth day of hospitalization. She was informed of the diagnosis of HIV infection, and referred to internal medicine for antiretroviral treatment. A thrombophilia test will be performed at the end of the anticoagulant treatment. An appointment is made in gynaecology for the management of the polymyomatous uterus.

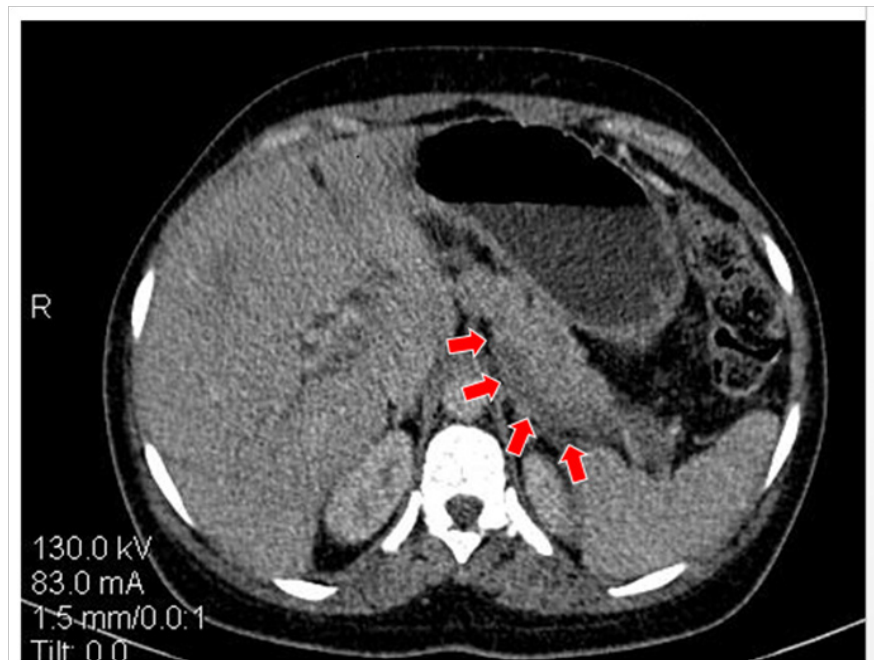

Figure 3 Abdominal-pelvic CT scan with portal time injection showing the extension of portal thrombosis to the splenic vein simulating peri- pancreatic oedematous infiltration.

\section{Discussion}

Portal vein thrombosis is a cruoric total or partial obstruction of the portal vein and/or one of its branches, right or left, more or less extended to the splenomesenteric system. ${ }^{4}$ It is a frequent complication of cirrhosis with an incidence that increases with the duration of the disease $\left(4.6 \%, 8.2 \%\right.$, and $10.7 \%$ respectively at 1,3 and 5 years) ${ }^{6}$ It has a rare occurrence when cirrhosis is not present with an incidence of 0.7/100,000 inhabitants in Europe..$^{2,3}$

The clinical picture is non-specific and depends on when the diagnosis is made. In the acute phase, the diagnosis is made in the case of abdominal pain with posterior irradiation either associated with fever or not. At a more advanced stage, the clinical manifestations of PVT are those of intestinal ischemia. In the chronic phase, the diagnosis is fortuitous or may be made in the presence of digestive bleeding, splenomegaly or other signs of portal hypertension. ${ }^{45,7}$ In our case, we think that the diagnosis was made in the acute phase of PVT due to the presence of acute transfixing epigastric pain in the back. These signs rarely suggest the diagnosis of portal vein thrombosis at the outset, which explains the diagnostic wandering observed in our case. Instead, these signs lead to abdominal imaging examinations, including ultrasound and/or abdominal CT scan, which are the key to diagnosis. ${ }^{8}$

Doppler ultrasound is often done as a first-line treatment to explore abdominal pain. In case of acute portal vein thrombosis, a hyperechoic thrombus, with an increase in the size of the portal vein and a lack of Döppler flow, can be seen at the very early stage. The signs in favour of chronic portal vein thrombosis are, in addition to morphological and hemodynamic abnormalities in the portal trunk, a network of collateral derivations (cavernoma portal). But such a cavernoma can develop in only a few weeks after an acute PVT (15 to 30days), and this has been observed in our patient. Indeed, we retain an acute thrombosis in our patient (despite the portal cavernoma) given the recent beginning of the symptomatology with intense pain, and the absence of esophageal varix. The abdominal CT scan with contrast injection, on the other hand, finds a hypodensity surrounded 
by the contrast agent. The diagnosis in our case was scanographic suggested by an increase in size and a hypodensity of the portal trunk, from the extended splenomesaraïque trunk to the splenic vein with the presence of portal cavernoma. It should be noted that in addition to the positive diagnosis of venous thrombosis, the $\mathrm{CT}$ also makes it possible to evaluate the extension of the thrombus, to look for a local cause and signs of intestinal ischemia. In rare cases, it may be necessary to perform an angio-MRI whose main interest is its ability to highlight biliary involvement in relation to cavernoma veins. ${ }^{4,5,7}$

Etiological factors in the absence of cirrhosis are represented by general prothrombotic disorders $(60 \%)$ and local factors $(30 \%)$. Similarly, a local factor must be sought even when a general prothrombotic disorder has been identified, especially since local and general factors are sometimes intertwined (15\%). The etiological factors found in our case were both local-regional (uterine myoma) and general (HIV infection). At the end of the etiological assessment, no cause can be found $(30 \%),{ }^{4,9}$ Among the general prothrombotic disorders is HIV infection. The incidence of thromboembolic venous disease among people living with HIV is 2 to 10 times higher than in the general population. ${ }^{10}$ The factors associated with this increase in incidence are:

a. The increase in life expectancy thanks to the advent of antiretrovirals,

b. A history of cytomegalovirus infection or other progressive opportunistic disease,

c. The use of anti-protease agents,

d. Acquired thrombophilia (acquired protein S deficiency),

e. A CD4 level of less than $200 / \mathrm{mm} 3^{11-13}$

The usual locations of thromboembolic venous disease among people living with HIV are represented by the veins of the pelvic limbs and the pulmonary artery. ${ }^{11}$ Rare locations such as portals have also been described and are sometimes indicative of HIV infection as in our case. ${ }^{12,14}$ Once the diagnosis has been made, active treatment is recommended to limit short and long-term complications. For both acute PVT and chronic thrombosis, the objective is to eliminate or reduce causal factors, limit thrombus extension and restore vascular permeability. Anticoagulation is the cornerstone of acute phase treatment and allows complete recanalization in $50 \%$ of patients after six months of treatment, partial recanalization in $40 \%$, with failure in $10 \%$. As with other venous thrombotic diseases, the effectiveness of anticoagulant therapy is largely dependent on how quickly it is implemented. In chronic PVT, the issue of anticoagulation must be weighed against the risk of bleeding from gastroesophageal varix and its role remains controversial (mainly symptomatic treatment). ${ }^{4,13}$

As with any thromboembolic venous disease, special attention must be paid to etiological treatment to prevent recurrence. ${ }^{4}$ The natural history of portal vein thrombosis diagnosed by modern imaging techniques is not well known. Previous studies of late diagnosed portal vein thrombosis reported high mortality from digestive hemorrhage. Recent studies have reported a relatively good prognosis. Mortality at 5 years of age is around $10 \%$. The majority of the deceased patients were over 60 years of age. In half of the patients who died, the cause of death was not related to portal vein thrombosis. Four main complications can occur. In the recent thrombosis stage, intestinal necrosis is the most serious complication, with mortality between 20 and $50 \%$ even when surgery has allowed resection of the infarcted intestinal segment. ${ }^{4,13}$ In the late stage of portal cavernoma, the main complication is digestive hemorrhage by rupture of esophageal varix (incidence around $25 \%$ patient/years in patients without preventive treatment). The 2 nd complication is the appearance of thrombosis outside the portal system (incidence around 6.5\% patients/year in the absence of anticoagulant treatment) and splenic or mesenteric infarction (incidence around 5\% patients/year in the absence of anticoagulant treatment). The last complication corresponds to biliary symptoms due to compression of the biliary tract by the tributaries of the cavernoma (with an incidence of around $2.5 \%$ patients/years). ${ }^{4,13}$

The evolution in our case was good with the regression of painful symptomatology and the absence of progressive complications.

\section{Conclusion}

Although non-specific, abdominal pain is the most common symptom of acute PVT. The diagnosis is based on Döppler ultrasound and/or abdominal CT scan. The presence of a local or regional factor should not exclude the search for general causes such as HIV infection.

\section{Acknowledgments}

None.

\section{Conflicts of interest}

Author declare sheer are no conflicts of interest.

\section{References}

1. Bayraktar Y, Harmanci O. Etiology and consequences of thrombosis in abdominal vessels. World J Gastroenterol. 2006;12(8):1165-1174.

2. Ageno W, Dentali F, Pomero F, et al. Incidence rates and case fatality rates of portal vein thrombosis and Budd-Chiari Syndrome. Thromb Haemost. 2017;117(4):794-800.

3. Rajani R, Bjornsson E, Bergquist A, et al. The epidemiology and clinical features of portal vein thrombosis: a multicentre study. Aliment Pharmacol Ther. 2010;32(9):1154-1162.

4. Meuwly JY, Saverot-Chevalier AL, Sgourdos G. Thrombose veineuse porte. Forum Med Suisse. 2012;12(6):119-125.

5. Condat B. Thrombosis of the portal vein Portal vein thrombosis Clinical and Biological Gastroenterology. 2006;30(10):1170-1176.

6. Nery F, Chevret S, Condat B, et al. Causes and consequences of portal vein thrombosis in 1,243 patients with cirrhosis : results of a longitudinal study. Hepatology. 2015;61(2):660-667.

7. Spahr LF, Froehlich A. How to manage for thrombosis in adults. Rev Med Suisse. 2002;2(2377).

8. Condat B, Valla D. Conduct to be held in front of thrombosis of the portal vein. Clinical and Biological Gastroenterology 1999;23:1210 1214.

9. Ponziani FR, Zocco MA, Campanale C, et al. Portal vein thrombosis: insight into physiopathology, diagnosis, and treatment. World J Gastroenterol. 2010;16(2):143-155.

10. Klein SK, Slim EJ, De Kruif, et al. Is chronic HIV infection associated with venous thrombotic disease? A systematic review. Neth $\mathrm{J}$ Med. 2005;63(4):129-136.

11. Ello FN, Bawe A, Kouakou GA, et al. Thromboembolic manifestations in 36 HIV-infected patients in West Africa. Pan Afr Med J. 2018;31:224. 
12. Ramanampamonjy RM, Ramarozatovo LS. Portal vein thrombosis in HIV-infected patients: report of four cases. The Internal Medicine Journal. 2005;26(7):545-548.

13. Mallet V, Viard JP, Pol S. Vascular diseases of the liver and HIV infection. The Infectiologist's Letter. 2011;26(1):14-17.
14. Konin C, Adoh M, Adoubi A, et al. Unusual venous thrombosis revealing a human immunodeficiency virus infection and a protein $\mathrm{S}$ deficiency. Two cases and literature review. The Internal Medicine Journal. 2008;29(6):508-511. 